\title{
Über das Verhalten freier und an Protoplasma gebundener Hefenenzyme. ${ }^{1}$ ) \\ Von
}

Hans Euler und Sixten Kullberg.

Mit sieben Figuren im Text.

(Aus dem biochemischen Laboratorium der Hochschule Stockholm.)

(Der Redaktion zugegangen am 18. Mai 1911.)

In bezug auf Wirksamkeit, Löslichkeit und Verhalten gegen anästhetisierende Mittel bestehen zwischen den kohlenhydratspaltenden Hefenenzymen, der Zymase (im weiteren Sinne), der Maltase und der Invertase erhebliche und anscheinend sehr wesentliche Unterschiede. Es soll in der vorliegenden Mitteilung gezeigt werden, daß die Versuchsergebnisse, welche hinsichtlich dieser drei Enzyme vorliegen, sich von einem gemeinsamen Gesichtspunkt aus darstellen lassen.

Wir geben zuerst eine Zusammenfassung der hierher gehörenden Tatsachen.

\section{Zymase.}

Lebende Hefezellen vergären Glukose unter folgenden Bedingungen. ${ }^{2}$ )

Die Gärung verläuft um so schneller, je verdünnter die Zuckerlösung ist. Nach Aberson verhält sich die Geschwindigkeit in $14 \%$ iger Glukoselösung zu der in $8,5 \%$ iger Lösung wie 64,4 zu 93,3. Nach Slator ${ }^{3}$ ) besteht ein Maximum der Geschwindigkeit in 4\% iger Lösung.

Damit steht in Zusammenhang, daß die Formel für Reaktionen erster Ordnung nicht zutrifft, sondern daß die nach

1) Z. T. aus Svenska Vet. Akad. Arkiv f. Kemi, Bd. 4, Nr. 13, 1911.

2) Rec. Trav. Chim. Pays-Bas, Bd. 22, S. 78, 1903.

s) Journ. Chem. Soc., Bd. 89, S. 133, 1906. 
dieser Formel berechneten Konstanten mit fortschreitender Reaktion wachsen. Aberson nimmt an, daß die Formel

$$
k=\frac{1}{t} \ln \frac{a+x}{a-x}
$$

für die Gärung allgemein gültig ist. Die Reaktionsgeschwindigkeit ist angenähert proportional der angewandten Hefemenge (l. c., S. 85).

In bezug auf die vorstehenden Resultate muß folgendes betont werden:

Aberson hat die Gärungsgeschwindigkeit auf optischem Wege, durch Beobachtung der Drehungsänderung der Zuckerlösung gemessen. Er hat angenommen, daß er damit die Mengen der bei der Reaktion verbrauchten Glukose ermittelt, und daß dieselben streng proportional sind mit der Menge der auftretenden Kohlensäure. Eine einfache Überlegung ${ }^{1}$ ) zeigt, daß dies nicht der Fall zu sein braucht, wenn man das Auftreten eines Zwischenproduktes berücksichtigt (Buchner und Meisenheimer), dessen Existenz zwar noch nicht bewiesen, aber wahrscheinlich geworden ist. Wenn nämlich ein Zwischenprodukt in meßbarer Konzentration auftritt, so kann dies nur dadurch geschehen, daß mehr Zucker gespalten wird, als der entwickelten Menge von Kohlensäure entspricht, und diese Abweichung von der Proportionalität zwischen verbrauchtem Zucker und gebildeter Kohlensäure muß sich so lange geltend machen, als ein Zwischenprodukt in der Lösung besteht. Wie Versuche, die der eine von uns mit Herrn Dr. Fodor ausgeführt hat, zeigen, tritt tatsächlich eine erhebliche Differenz auf zwischen dem Rückgang der optischen Drehung einerseits und der entwickelten Kohlensäuremenge anderseits. Die Versuche von Aberson liefern also kein vollständiges Bild der Gärungsgeschwindigkeit; vielmehr geben sie, unter Voraussetzung, daß das Zwischenprodukt der Gärung optisch inaktiv ist, die Reaktionsgeschwindigkeit: Zucker - Zwischenprodukt. Dieser Vorgang wird zweifellos sekundär durch den weiteren Zerfall des

1) H. Euler und B. af Ugglas, Zeitschrift f. allgem. Physiologie, Bd. 12, S. 364; 1911. 
Zwischenproduktes beeinflußt. Mißt man die Entwickelung der Kohlensäure, so bestimmt man dadurch die Geschwindigkeit:

$\mathrm{Zwischenprodukt} \rightarrow$ Alkohol $+\mathrm{CO}_{2}$, welche ihrerseits durch die Bildungsgeschwindigkeit des Zwischenproduktes beeinflußt wird.

Es ist also von vornherein nicht zu erwarten, daß die nach den bisher angewandten Methoden gemessene Zuckerspaltung durch lebende Hefe, Dauerhefe oder Hefepreßsaft sich als einfache Reaktion erster Ordnung darstellen läßt.

Einfluß von Toluol, Thymol und Chloroform auf die Gärung der lebenden Hefe.

Die Gärung der lebenden Hefe wird durch anästhetisierende Mittel schnell und fast vollständig aufgehoben. Jedoch trat bei unserer Hefe anfangs eine, allerdings geringe, Kohlensäureentwickelung ein.

$1 \mathrm{~g}$ abgepreßte Hefe $+20 \mathrm{ccm} 10 \%$ iger Rohrzuckerlösung. Ohne Chloroform Mit $1 \mathrm{ccm}$ Chloroform Mit Thymol $\mathrm{g} \mathrm{CO}_{2}$ in 120 Minuten $0,2038 \quad 0,0105 \quad 0,0110$

Die geringfügige, aber immerhin meßbare Kohlensäureentwicklung, die in Gegenwart von Chloroform und Thymol eintrat, blieb auch dann nicht aus, wenn die Hefe vor dem Zuckerzusatz mit dem betreffenden Antiseptikum behandelt wurde.

$0,25 \mathrm{~g}$ Hefe $+25 \mathrm{ccm} \mathrm{H}_{2} \mathrm{O}+1 \mathrm{ccm}$ Chloroform. Bleibt nach dem Umschütteln 30 Minuten stehen. Hierauf Zusatz von $4 \mathrm{~g}$ Rohrzucker.

$$
\begin{aligned}
& \text { g Kohlensäure } \\
& \text { Nach } 38 \text { Minuten. . . . . . 0,011ら } \\
& \text { Nach weiteren } 28 \text { Stunden . . } 0,0125
\end{aligned}
$$

Auch Toluol übt eine ähnliche Wirkung aus, wie die beiden folgenden Versuchspaare zeigen. (Fig. 1 a.)

I. $0,5 \mathrm{~g}$ abgepreßte Hefe $+2 \mathrm{~g}$ Glukose $+25 \mathrm{ccm}$ $2 \%$ iger $\mathrm{NaH}_{2} \mathrm{PO}_{4}$.

a) Ohne Toluol

b) Mit $2 \mathrm{ccm}$ Toluol. Wasser.

II. $0,5 \dot{\mathrm{g}}$ abgepreßte Hefe $+2 \mathrm{~g}$ Glukose $+25 \mathrm{ccm}$

a) Ohne Toluol

b) Mit $2 \mathrm{ccm}$ Toluol. 
In Gegenwart von Toluol bleibt also höchstens etwa $1 \%$ der Gärwirkung erhalten, auch dieser Rest verschwindet nach einigen Stunden.

\section{Einfluß von $\mathrm{NaH}_{2} \mathrm{PO}_{4}$.}

Aus der Figur 1 a geht gleichzeitig hervor, daß die Gärung unserer Hefe $\mathrm{H}$ durch $20 \%$ iges $\mathrm{NaH}_{2} \mathrm{PO}_{4}$ beschleunigt wird und zwar um etwa $25 \%$. Es entspricht dies früher von uns an der gleichen Hefe gewonnenen Ergebnissen.

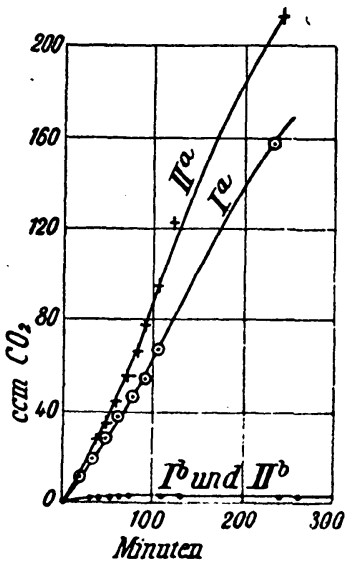

Fig. 1 .

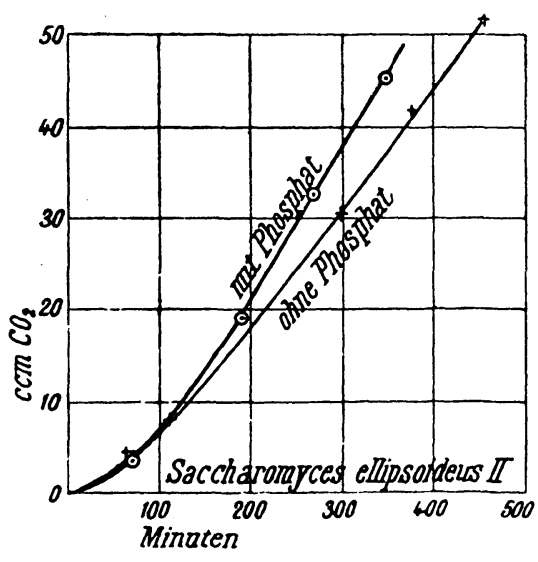

Fig. 1b.

Wir haben auch einige Versuche über den Einfluß von $\mathrm{NaH}_{2} \mathrm{PO}_{4}$ auf andere Hefearten angestellt.

Versuch mit Saccharomyces ellipsoïdeus.

0,5 g Saccharomyces ellipsoïdeus II (abgepreßt, drei Monate alte Kultur aus mit Rohrzucker versetzter Nährungslösung) $+25 \mathrm{ccm} 8 \%$ iger Glukoselösung. Im Parallelversuch war die Lösung $2 \%$ ig in bezug auf $\mathrm{NaH}_{2} \mathrm{PO}_{4}$. Siehe Fig. $1 \mathrm{~b}$.

Die Beschleunigung durch das saure Phosphat erwies sich hier etwas geringer als bei unserer Bierhefe $\mathrm{H}$.

Versuch mit Saccharomyces thermantitonum.

Die Hefe war in Hefewasser kultiviert worden unter $\mathrm{Zu}$ satz von $1 \%$ Glukose. 
$0,5 \mathrm{~g}$ Hefe $+25 \mathrm{ccm}$ $8 \%$ ige Glukoselösung. Im Parallelversuch war die Lösung $2 \%$ ig in bezug auf $\mathrm{NaH}_{2} \mathrm{PO}_{4}$. Siehe Fig. $1 \mathrm{c}$.

Die Beschleunigung ist etwa die gleiche wie bei unserer Bierhefe $\mathrm{H}$.

Gärung durch Trockenhefe.

Beim Trocknen bleibt bekanntlich ein Teil der Zymase aktiv, auch wenn die so ge-

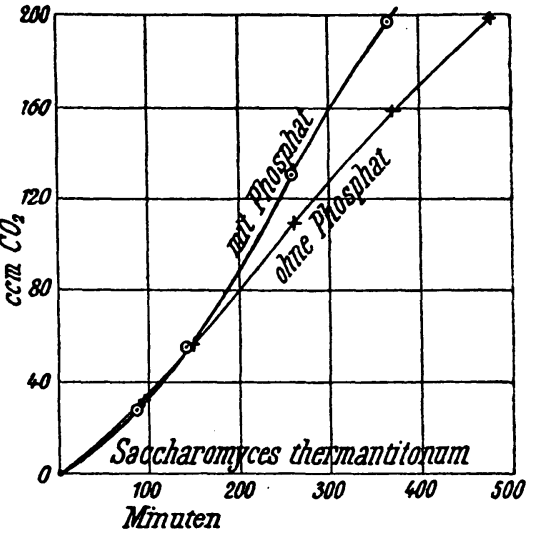

Fig. $1 \mathrm{c}$. wonnenen Dauerpräparate ganz steril sind. Da keine Angaben darüber vorliegen, welcher Anteil der Gärwirkung sich bei vorsichtigem Entwässern erhalten läßt, ${ }^{1}$ ) geben wir hier einige Versuche an.

Bei denselben kam die Hefe teils frisch abgepreßt zur Anwendung, teils nach dem Trocknen. Die Trocknung geschah im Vakuum unter $40^{\circ}$; hierauf folgte eine etwa halbstündige Erhitzung auf etwa $90^{\circ}$.

$0,25 \mathrm{~g}$ abgepreßte Hefe in $25 \mathrm{ccm} 8 \%$ iger Glukoselösung :

Ungetrocknet Nach dem Trocknen Verhältnis

$\begin{array}{lllll}\text { g } \mathrm{CO}_{2} \text { nach 4 Stunden } & 0,0620 & 0,0038 & 16 \\ \text { > } 20 & 0,3254 & 0,0090 & 36\end{array}$

$1 \mathrm{~g}$ abgepreßte Hefe in $20 \mathrm{ccm} 10 \%$ iger Rohrzuckerlösung:

Ungetrocknet Nach dem Trocknen Verhältnis

Vers. a) $\mathrm{g} \mathrm{CO}_{2}$ nach 2 Stunden 0,3200

, b) $>2>0,1937$

c) $>$, 2 , 0,1939

0,0110

0,0140

0,0140

$30: 1$

$14: 1$

1) Quantitative Untersuchungen hat Buchn er (Zymasegärung, S.252) nur in der Weise angestellt, daß einerseits die Gärkraft des Preßsaftes eines Trockenpräparates mit dem Preßsaft aus frischer Hefe verglichen wurde, wobei natürlich die unvermeidlichen Versuchsfehler groß sein müssen. 
$1 \mathrm{~g}$ abgepreßte Hefe (2-3 Stunden vorbehandelt mit neutralisiertem $\mathrm{KH}_{2} \mathrm{PO}_{4}$ ) in $20 \mathrm{ccm} 10 \%$ iger Rohrzuckerlösung :

Ungetrocknet Nach dem Trocknen Verhältnis

Vers. a) $\mathrm{g} \mathrm{CO}_{2}$ nach 2 Stunden 0,2345

0,0087

$27: 1$

$\triangleright \mathrm{b}),>2,0,2236$

0,0094

$25: 1$

$1 \mathrm{~g}$ abgepreßte Hefe (2-3 Stunden vorbehandelt mit

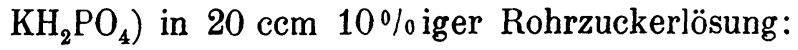

Ungetrocknet Nach dem Trocknen Verhältnis

Vers. a) $\mathrm{g} \mathrm{CO}_{2}$ nach 2 Stunden 0,2231

0,0137

$16: 1$

, b) $>>2,0,2395$

0,0172

$14: 1$

Bei sehr vorsichtiger Entwässerung durch Trocknung ließ sich also höchstens $1 / 10-1 / 30$ der Zymase wirksam erhalten.

Bei der Entwässerung unserer Hefe mit Alkohol und Äther wurde die Gärwirkung noch mehr geschwächt als durch das Trocknen im Vakuum.

Eine Tötung der Hefe wurde auch bei der Temperatur der flüssigen Luft vorgenommen.

2 verschlossene Kölbchen mit je $0,5 \mathrm{~g}$ abgepreßter Hefe (Wassergehalt 62\%) wurden während einer halben Stunde in ein Bad von flüssiger Luft gestellt. Dann wurden die Kölbchen auf Zimmertemperatur gebracht und gleichzeitig mit 2

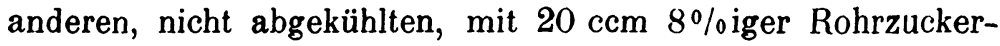
lösung versetzt. Die Kohlensäureentwicklung wurde volumetrisch verfolgt.

Tabelle 1.

\begin{tabular}{c|c|c}
\hline \multirow{2}{*}{ Minuten } & \multicolumn{2}{|c}{$\mathrm{ccm} \mathrm{CO}_{2}$} \\
& gefrorene Hefe & nicht gefrorene Hefe \\
\hline 50 & 1 & 31 \\
100 & 2 & 62 \\
200 & 5 & 127
\end{tabular}

Durch eine halbstündige Abkühlung auf etwa $-180^{\circ}$ wird also die Hefe stark geschwächt, aber nicht vollkommen getötet. 


\section{Anästhetisierende Mittel.}

Durch mechanisches Zerreiben der Hefezellen mit Sand und Kieselgur ist es bekanntlich Buchner gelungen, einen zellfreien, gärungskräftigen Saft darzustellen. Die Gärwirkung dieses Saftes wird durch Zusätze von Toluol fast nicht, durch Thymol und Chloroform wenig beeinflußt.

Es wäre demnach zu erwarten gewesen, daß auch die Gärkraft der Trockenhefe durch die genannten antiseptischen Stoffe nur wenig vermindert wird. Indessen zeigte sich eine auffallend starke Beeinflussung.

I. Thymol. Bierhefe im Passburgschen Vakuumapparat unter $40^{\circ}$ vollständig entwässert. Dann während 2 Stunden bei ansteigender Temperatur $\left(50-100^{\circ}\right)$ erhitzt.

$0,25 \mathrm{~g}$ dieser Dauerhefe $+20 \mathrm{ccm} 10 \%$ iger Rohrzuckerlösung :

$$
+5 \mathrm{ccm} \text { Wasser } \quad+5 \mathrm{ccm} \text { Thymolwasser }
$$

(gesättigt)

Menge $\mathrm{CO}_{2}$ in 14 Stunden $\quad 0,0080 \mathrm{~g}$ $0,0026 \mathrm{~g}$

II. Toluol. $1 \mathrm{~g}$ Trockenhefe $+25 \mathrm{ccm} 8 \%$ iger Glukoselösung $+2 \mathrm{ccm}$ Toluol bei $30,6^{\circ}$. (Siehe folgende Figur 2.)

Die Einwirkung des als «Gift * wirkenden Toluols macht sich vom Beginn der Reaktion an geltend, der Vergiftungsgrad ist aber nicht konstant, sondern nimmt mit fortschreitender Reaktion zu.

Da die im Vakuum getrocknete Hefe selten ganz steril ist und also die Möglichkeit vorlag, daß ein nicht zu vernachlässigender Anteil des Hefepräpa-

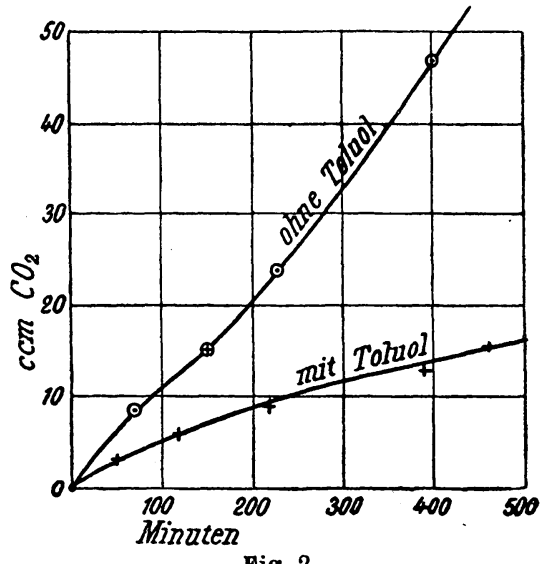

Fig. 2. rates noch lebensfähige Zellen enthielt, so haben wir dieses Präparat während einer halben Stunde mit absolutem Alkohol behandelt. Nach dem Abpressen und Trocknen wurde dann ein 
Versuch ganz in der gleichen Weise angestellt wie der zuletzt beschriebene. Das Ergebnis desselben geht aus den Kurven der Fig. 3 hervor. Toluol übt also auch auf vollkommen sterile Trockenhefe eine stark hemmende Wirkung aus.

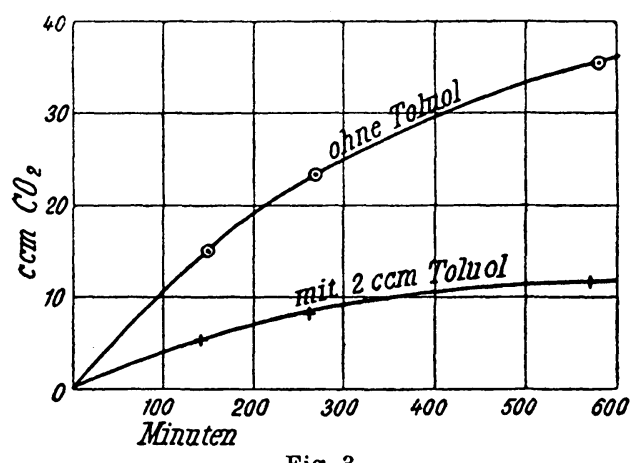

Fig. 3.

II. Maltase.

In bezug auf die Maltosespaltung durch Hefe sind die Ergebnisse verschiedener Untersuchungen sehr auseinandergegangen. Die genauesten Angaben verdanken wir E. Fischer. ${ }^{1}$ ) Feuchte, unverletzte Hefe spaltet in Gegenwart von viel Chloroform- $\alpha$-Methylglukosid; in Chloroformwasser betrug die Glukosidspaltung durch Saaz-Hefe $25 \%$, während eine Frohberg-Hefe keine Spaltung bewirkte. Maltose wird in Chloroformwasser von keiner lebenden Hefe gespalten. In Gegenwart anderer Antiseptika trat sowohl in Maltoselösungen als in $\alpha$-Methylglukosidlösungen Spaltung ein.

Nach eigenen Versuchen mit Unterhefe der hiesigen St. Eriks-Brauerei verhält sich die lebende Hefe gegenüber Maltose folgendermaßen:

$0,5 \mathrm{~g}$ Hefe in $25 \mathrm{ccm} 8 \%$ iger Maltoselösung: Reaktionszeit: 260 Minuten bei $30^{\circ}$.

Drehungsrückgang

Ohne Toluol . . . 3,08 ${ }^{\circ}=31,0 \%$ Mit $1 \mathrm{ccm}$ Toluol $\cdot 0,41^{\circ}=4,2 \%$
Kohlensäureentwicklung

$$
0,1990=20,0 \%
$$$$
0,0103=1,0 \%
$$

Frische Hefe spaltet aber Maltose je nach Art und Vorbehandlung. Bei einem Versuch mit sehr kräftiger Bierhefe, welche ich selbst in Pasteurschen-Kolben auf mit Maltose versetztem Hefewasser gezüchtet habe, verlief die Maltosespaltung anscheinend etwas schneller als die Gärung. In den

1) Ber. d. Deutsch. chem. Ges., Bd. 28, S. 1429, 1895. 
meisten Fällen aber waren die Resultate zweifelhaft. Wir führen beispielsweise folgenden Versuch an:

In $25 \mathrm{ccm} 8 \%$ iger Maltoselösung $1 \mathrm{~g}$ abgepreßte Hefe. Reaktionszeit: 5 Stunden bei $20^{\circ}$.

$\begin{array}{lc}\text { Drehungsrückgang } & \text { Kohlensäureentwicklung } \\ \text { Ohne Toluol . . . . . } 4,10^{\circ}=42,0 \% & 0,2643 \mathrm{~g}=27,0 \% \\ \text { Mit } 1 \mathrm{ccm} \text { Toluol . . . } 0,52^{\circ}=5,3 \% & 0,0170 \mathrm{~g}=1,7 \%\end{array}$

Nun betragen zwar in den beiden letzten Versuchen die entwickelten Mengen $\mathrm{CO}_{2} 20$ und 27 bezw. 1 und 1,7\% der bei vollständiger Reaktion auftretenden Kohlensäure, während der Drehungsrückstand 31 und 42 , bezw. 4,2 und $5,3 \%$ beträgt. Indessen zeigt sich eine derartige Differenz auch stets bei der Vergärung der Glukose und beruht, wie in einer folgenden Mitteilung näher gezeigt werden wird, auf der Bildung eines Zwischenproduktes. ${ }^{1}$ )

In Übereinstimmung mit Morris ${ }^{2}$ ) und mit E. Fischer fand ich, daß Chloroform die Maltosespaltung ebenso vollständig aufhebt wie die Gärung. Bei Gegenwart von Toluol, Äther und anderen antiseptischen Mitteln findet nach Fischer Maltosespaltung statt. Immerhin wird schon durch Zusatz von Toluol die Maltosespaltung der lebenden. Hefe sehr stark herabgedrückt, wie z. B. obiger Versuch zeigt.

In bezug auf Dauerhefe gibt Fischer an: *War die Hefe trocken, so geht die Hydrolyse des Disaccharides (der Maltose) leicht vonstatten. Unter diesen Umständen wird eben das Enzym gelöst, wovon ich mich durch einen besonderen Versuch mit gesättigtem Chloroformwasser überzeugt habe.»

\section{Invertase.}

Die chemische Dynamik der Rohrzuckerspaltung durch lebende Hefe haben wir in einer vorhergehenden Mitteilung studiert. Diese Zeitschrift, Bd. 71, S. 14, 1911.

Durch Wasser läßt sich Invertase aus frischen Hefezellen bei gewöhnlicher Temperatur bekanntlich nur in sehr geringem

1) Proc. Chem. Soc., 1895, S. 46.

2) H. Euler und A. Fodor, Svenska Vet. Akad. Arkiv f. Kemi, Bd. 4, 1911. 
$\mathrm{Maß}$ auslaugen; schon bei $50^{\circ}$ kann aber aus frischer Hefe viel aktive Invertase direkt extrahiert werden. Noch vollständiger ist die Extraktion, wenn die Hefe nach den bekannten Verfahren vor der Extraktion entwässert wird.

Wir haben einen hierauf bezüglichen quantitativen Versuch angestellt. ${ }^{1}$ )

Es lagen bis jetzt keine Angaben darüber vor, wie viel von der invertierenden Fähigkeit der Hefe erhalten bleibt, wenn frische Hefe vorsichtig im Vakuum getrocknet wird.

$0,25 \mathrm{~g}$ lebende, abgepreßte Hefe in $25 \mathrm{ccm} \mathrm{10 \%}$ iger Rohrzuckerlösung bei $20^{\circ}$. Wassergehalt der Hefe: 57,3\%.

\section{Tabelle 2.}

\begin{tabular}{c|r|c|c}
\hline Minuten & Drehung & $\mathrm{a}-\mathrm{x}$ & $\mathrm{k} \cdot 10^{4}$ \\
\hline 0 & 4,07 & 5,37 & - \\
15 & 1,38 & 2,68 & 201 \\
25 & 0,45 & 1,75 & 195 \\
35 & $-0,03$ & 1,27 & 180 \\
$\infty$ & $-1,30$ & - & -
\end{tabular}

$0,1 \mathrm{~g}$ getrocknete Hefe in $25 \mathrm{ccm} 10 \%$ iger Rohrzuckerlösung bei $20^{\circ}$.

1) $5 \mathrm{~g}$ Trockenhefe wurden während 12 Stunden mit $100 \mathrm{ccm}$ Wasser extrahiert. $5 \mathrm{ccm}$ dieses Extraktes wurden mit $20 \mathrm{ccm} 10 \%$ iger Rohrzuckerlösung und $5 \mathrm{ccm} 5 \%$ iger $\mathrm{NaH}_{2} \mathrm{PO}_{4}$-Lösung gemischt. Mit dieser Lösung wurde die Inversionskonstante

$$
\mathrm{k} \cdot 10^{4}=48
$$

erhalten. Dies entspricht einer extrahierten Hefemenge von $0,25 \mathrm{~g}$. Nach obiger Tabelle würde die entsprechende Menge getrockneter Hefe in $25 \mathrm{ccm}$ der gleichen Rohrzuckerlösung eine Inversionskonstante

$$
\mathrm{k} \cdot 10^{4}=220
$$

liefern. Durch Extraktion der Trockenhefe mit dem 20-fachen Gewicht Wasser geht also etwa ein Viertel ihres Invertasegehaltes in Lösung.

Da ferner beim Trocknen der Hefe etwa die Hälfte der invertierenden Wirkung erhalten bleibt (siehe unten), so ergibt sich, daß nach der üblichen Extraktionsmethode etwa 1/8 des Invertasesystems in die wässerige Lösung übergeht. 
Tabelle 3.

\begin{tabular}{c|r|c|c}
\hline Minuten & Drehung & $\mathrm{a}-\mathrm{x}$ & $\mathbf{k} \cdot 10^{4}$ \\
\hline 0 & 4,07 & 5,37 & - \\
17 & 2,41 & 3,71 & 94 \\
26 & 1,81 & 3,21 & 86 \\
37 & 1,32 & 2,62 & 84 \\
$\infty$ & $-1,30$ & - & -
\end{tabular}

Rechnet man die Konstanten auf Grund des oben angegebenen Wassergehaltes auf gleiche Hefemengen um, so ergibt sich:

$$
\begin{array}{lc} 
& \mathrm{k} \cdot 10^{4} \\
\text { Frische Hefe. . . . } 192 \\
\text { Getrocknete Hefe. . . } 88
\end{array}
$$

Es bleibt also etwa die Hälfte der Invertase beim Entwässern erhalten. Zum Vergleich sei erwähnt, daß die gleiche Hefe bei derselben Trocknung nicht mehr als etwa 1/20 ihrer Gärwirkung behielt.

Zusatz von Chloroform, Toluol und Thymol ist fast ganz ohne Einfluß auf die invertierende Wirkung der Hefe. Dies geht aus folgendem Versuch hervor, der mit 10\% iger Rohrzuckerlösung bei $20^{\circ}$ angestellt worden ist.

$$
\begin{aligned}
& \mathrm{k} \cdot 10^{4} \\
& \text { Mit Chloroform .. } 94 \\
& \text { Ohne » .. } 96
\end{aligned}
$$

Ebenso ist die Invertase der getrockneten Bierhefe gegen Zusatz von Giften unempfindlich.

Anders als in gewöhnlicher Hefe verhält sich die Invertase in Monilia candida. Dieselbe ist ebenfalls von E. Fischer studiert worden. Er gibt an: «Frische Monilia spaltet in Gegenwart von antiseptischen Mitteln Rohrzucker nicht. Trocknet man die Moniliazellen, so tritt auch in Gegenwart von antiseptischen Stoffen Spaltung ein».

Die obigen Ergebnisse stellen wir jetzt in einer Tabelle zusammen. 
Tabelle 4 .

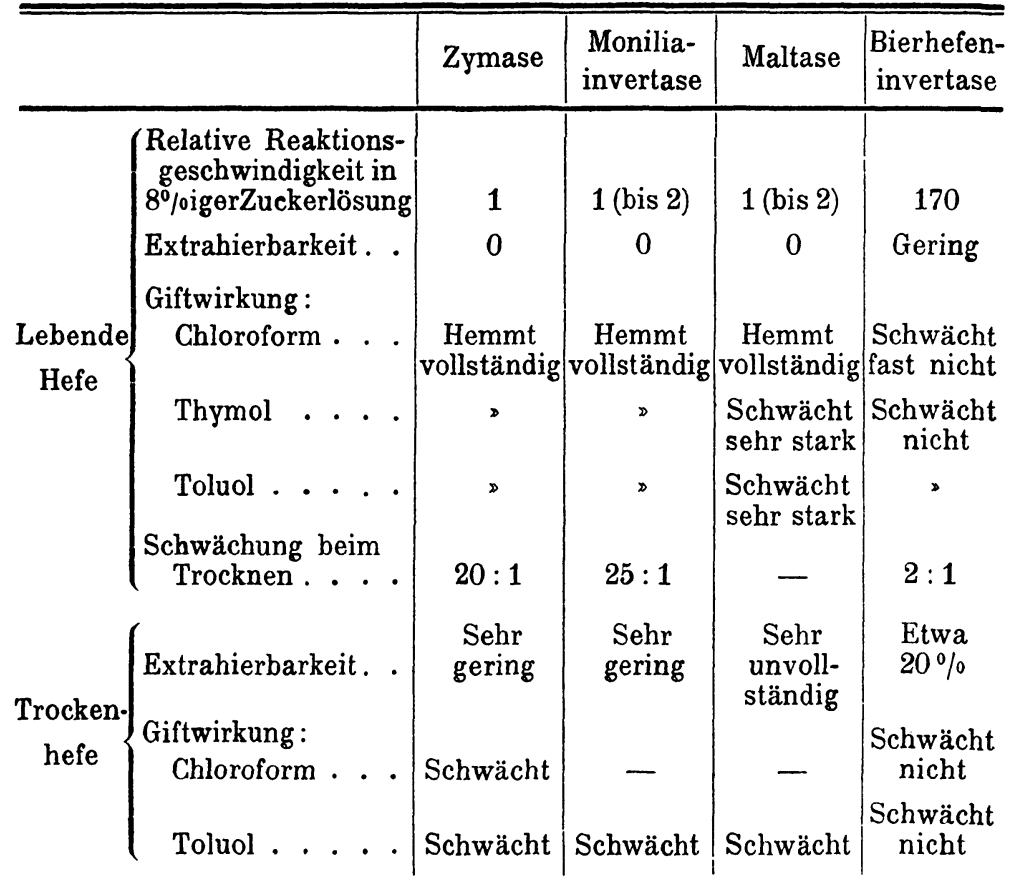

In erster Linie fällt in vorstehender Tabelle der kontinuierliche Übergang im Verhalten der verschiedenen Hefenenzyme auf.

Hinsichtlich der «Zymase» wurde früher der Schluß gezogen, daß dieselbe (zum größten Teil) mit dem Protoplasma verbunden ist, und von demselben durch Entwässern mehr oder weniger vollständig abgetrennt wird. Nun lassen sich die über die Hefenenzyme oben mitgeteilten Tatsachen durch folgende erweiterte Arbeitshypothese zusammenfassen:

Die Hefenenzyme sind ursprünglich Bestandteile des Plasmas und werden entweder schon in der lebenden Zelle vom Plasma abgeschieden und dann am Plasma wieder regeneriert; sie sind dann relativ leicht extrahierbar und sind in relativ großer Menge in den Zellen vorhanden. Oder aber die Abtrennung erfolgt erst (teilweise) beim Entwässern der Hefe oder durch mechanische 
Mittel, überhaupt unter den Umständen, unter welchen das Plasma getötet wird. Gegen Antiseptika sind die Hefenenzyme in dem Maße unempfindlich, als sie vom lebenden Plasma befreit sind.

Hierzu sind folgende Erläuterungen zu fügen:

Man könnte einwenden, daß die «Zymase» der Dauerhefe, welche vom lebenden Plasma befreit ist, sich etwa im selben Grad wie die «Maltase» extrahieren lassen sollte. Dies scheint tatsächlich der Fall zu sein. Ich ließ sorgfältig getrocknete Brauereihefe mit der doppelten Menge Wasser 24 Stunden stehen und filtrierte dann. Das Filtrat zeigte deutliche, wenn auch geringe Gärung. Aus $20 \mathrm{ccm}$ Filtrat entwickelten sich bei $30^{\circ}$ in 24 Stunden $10 \mathrm{ccm} \mathrm{CO}_{2}$. Bei anderen Hefen gelingt die Extraktion vielleicht besser. ${ }^{1}$ )

Die in obiger Tabelle angegebenen Reaktionsgeschwindigkeiten können natürlich in verschiedenen Hefen schwanken; es sind Mittelwerte, welche nur zur allgemeinen Orientierung dienen sollen. Auch ist die Größe dieser Reaktionsgeschwindigkeit kein genaues Maß für die Enzymmengen, da ja die verschiedenen Reaktionen bei gleicher Katalysatormenge verschieden rasch verlaufen. Immerhin zeigt sich deutlich, daß in der gewöhnlichen Bierhefe viel mehr Invertase als Maltase vorhanden ist.

Bei Monilia liegen die Verhältnisse anders: Hier bleibt das Enzym in der lebenden Zelle an das Plasma gebunden, wird infolge dessen nicht regeneriert und die invertierende Wirkung ist demgemäß sehr gering (anderseits ist das Enzym fast nicht extrahierbar und wird durch Antiseptika gehemmt). Die unbedeutende invertierende Wirkung der Moniliazellen geht aus folgenden quantitativen Versuchen hervor: Die Hefe entstammt dem Berliner «Institut für Gärungsgewerbe» und wurde hier auf Hefewasser unter Maltosezusatz gezüchtet; es kam eine 14 Tage alte Kultur zur Verwendung.

1) Seit der Ausführung dieser im Februar 1911 veröffentlichten Versuche ist A. v. Lebedew zu ähnlichen Ergebnissen gekommen (Compt. rend., Bd. 152, S. 49, 1911). Wir verweisen auf die interessante Mitteilung dieses Forschers. 
Sämtliche Zuckerlösungen sind $8 \%$ ig und enthalten $0,5 \mathrm{~g}$ frische Moniliahefe in $25 \mathrm{ccm}$. Temp. $30^{\circ}$.

Wassergehalt der Hefe: 60,3\%.

Tabelle 5 .

\begin{tabular}{|c|c|c|c|c|}
\hline & $\begin{array}{c}\text { Reaktions- } \\
\text { zeit } \\
\text { Minuten }\end{array}$ & Drehung & $\begin{array}{l}\text { Drehungs- } \\
\text { rückgang }\end{array}$ & $\begin{array}{c}\text { Entwickelte } \\
\qquad \mathrm{CO}_{2}\end{array}$ \\
\hline Glukose . . . . & 0 & $4,34^{0}$ & & \\
\hline ..... & 187 & $3,43^{\circ}$ & $0,91^{\circ}=20,97 \%$ & $0,0660 \mathrm{~g}=6,60^{\circ}$ \\
\hline$\therefore .$. & 0 & $9,90^{\circ}$ & & \\
\hline . . . . & 185 & $9,50^{\circ}$ & $0,40^{\circ}=6,55^{\circ} \%$ & $0,0220 \mathrm{~g}=2,20 \%$ \\
\hline Saccharose . . . & 0 & $5,08^{\circ}$ & & \\
\hline .... & 181 & $4,71^{\circ}$ & $0,37^{\circ}=5,51 \%$ & $0,0400 \mathrm{~g}=4,0 \%$ \\
\hline andere & 0 & $5,08^{0}$ & & \\
\hline - Moniliakultur & 180 & $4,65^{\circ}$ & $0,43^{\circ}=6,41^{\circ} \%$ & $0,0500 \mathrm{~g}=5,0^{\circ} \%$ \\
\hline Mycoderma & 0 & $5,08^{\circ}$ & & \\
\hline $\begin{array}{c}\text { der gleicnen } \\
\text { Kultur }\end{array}$ & 200 & $4,65^{\circ}$ & $0,43^{\circ}=6,41 \%$ & $0,05 \check{0} 0 \mathrm{~g}=5,5 \%$ \\
\hline
\end{tabular}

Von Monilia wird also Glukose am schnellsten vergoren, Maltose am langsamsten. Von gewöhnlicher Bierhefe wird im allgemeinen Maltose etwa ebenso schnell vergoren wie Glukose und Rohrzucker.

In einer vorhergehenden Mitteilung ${ }^{1}$ ) wurde gezeigt, daß die Gärung lebender Hefe durch Vorbehandlung mit verdünnter ( $2 / 3-1 \%$ iger reiner oder neutralisierter) Monophosphatlösung nicht wesentlich beeinträchtigt wird, während die Vorbehandlung mit stärkeren Monophosphatlösungen eine deutliche Schwächung hervorruft.

Wird Trockenhefe mit $2 \%$ iger $\mathrm{KH}_{2} \mathrm{PO}_{4}$-Lösung vorbehandelt, so zeigt sich gegenüber einem mit Wasser digerierten Präparat eine deutliche Schwächung.

\section{Versuch:}

1. $10 \mathrm{~g}$ Trockenhefe wurden während 3 Stunden mit $100 \mathrm{ccm}$ Wasser digeriert, abgesogen und auf Ton getrocknet.

1) H. Euler u. S. Kullberg, Diese Zeitschrift, Bd. 71, S. 14, 1911. 
2. $10 \mathrm{~g}$ Trockenhef e wurden während 3 Stunden mit $100 \mathrm{ccm} 2 \%$ iger $\mathrm{KH}_{2} \mathrm{PO}_{4}$-Lösung behandelt. Filtration und Trocknen wie bei 1 .

Je $1 \mathrm{~g}$ der nach 1 und 2 vorbehan-

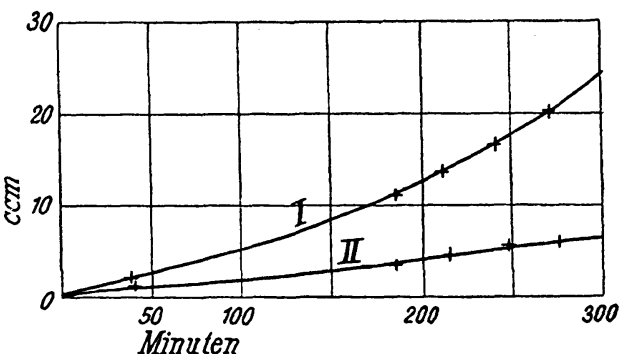

Fig. 4.

delten Hefe wurden mit $25 \mathrm{ccm} 8 \%$ iger Glukoselösung versetzt. Gärungstemperatur $30,6^{\circ}$.

Durch Vorbehandlung unserer Hefe mit Rohrzuckerlösungen wird die weitere Gärkraft der Hefe nicht beeinflußt. Gärt hingegen die Hefe in Gegenwart von Phosphat, so zeigt sich dann die Gärkraft unserer Hefe verstärkt. ${ }^{1}$ )

Es kann angenommen werden, daß hier der günstige Einfluß des Phosphates mit dem konstanten Phosphatgehalt in der Hefe zusammenhängt. Wir haben in folgenden Versuchen die Hefe in Gegenwart anderer Bestandteile ihrer Asche, nämlich in Gegenwart von $\mathrm{Mg}-$, $\mathrm{Ca}-$ und $\mathrm{SO}_{4^{-}}$ Ionen, vergären lassen.

Versuche: $30 \mathrm{~g}$ Hefe $\mathrm{H}$ vergären während 2 Stunden $300 \mathrm{ccm}$ einer 10\% igen Rohrzuckerlösung, unter Zusatz von:

I. $1,5 \mathrm{~g} \mathrm{MgSO}_{4}$,

II. $1,5 \mathrm{~g} \mathrm{CaCl}_{2}$,

III. $\mathrm{CaSO}_{4}$ (gesättigte Lösung).

Der Parallelver'such ohne Zusatz ist mit IV bezeichnet.

Das Resultat geht aus den Kurven der Fig. 5 hervor. Von den drei Salzen beschleunigte nur

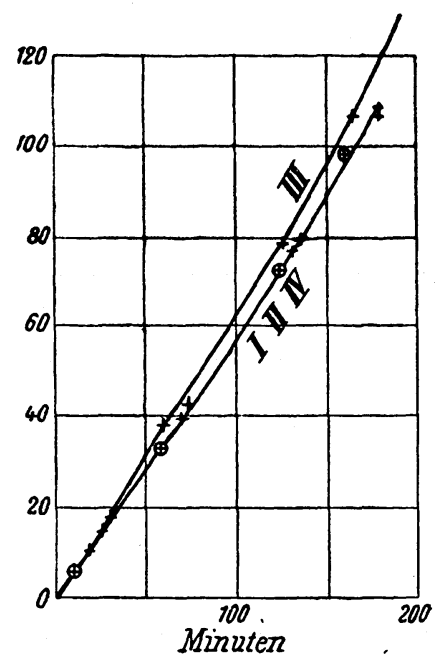

Fig. 5.

1) Euler und Lundeqvist, Diese Zeitschrift, Bd. 72, 1911.

Vgl. hierzu die zahlreichen Versuche von Lange (Woch. f. Brauerei, 1907). 
100 H. Euler und S. Kullberg, Über das Verhalten von Hefenenzyme.

das $\mathrm{CaSO}_{4}$ die Gärung. Wird eine $10 \%$ ige Glukoselösung durch $0,5 \mathrm{~g}$ abgepreßte Hefe $\mathrm{H}$ bei $30^{\circ}$ mit der Geschwindigkeit 1 vergoren, 1 ) so beträgt die Gärungsgeschwindigkeit, wenn die gleiche Lösung $2 \%$ ig in bezug auf $\mathrm{NaH}_{2} \mathrm{PO}_{4}$ ist, $\mathrm{k}=1,4$.

Wird die gleiche Hefe getrocknet, so ist die Beschleunigung erheblich größer, wie der folgende Versuch zeigt; bei welchem die Lösung a in bezug auf $\mathrm{NaH}_{2} \mathrm{PO}_{4} 1 \%$ ig war, während die Lösung $b$ kein Phosphat enthielt.

Unmittelbar nach der letzten Ablesung wurde die Drehung der Lösung ermittelt, um die Menge des in der Zeit $t$ verschwundenen Zuckers mit der Menge der in der gleichen Zeit entwickelten Kohlensäure zu vergleichen. Solche Vergleiche hat der eine von uns mit lebender Hefe angestellt; sie gaben einen Aufschluß über die Menge der in der Lösung anwesenden 'Zwischenprodukte. ${ }^{2}$ )

Tabelle 6.

\begin{tabular}{c|r|r|r|r|r|r|r|r|r|}
\hline \multirow{2}{*}{ Minuten } & \multicolumn{2}{|c|}{$\mathrm{ccm} \mathrm{CO}_{2}$} & \multicolumn{2}{|c|}{ In \% der } & \multicolumn{3}{|c|}{ Drehung } & \multicolumn{2}{|c|}{ Drehungsänderung } \\
in \% \%
\end{tabular}

Die Differenz zwischen der entwickelten $\mathrm{CO}_{2}$ und dem umgewandelten Zucker ist hier auffallenderweise sehr viel geringer als bei der Gärung mit lebender Hefe, wo zuweilen die Drehungsänderung das Doppelte des aus den entwickelten Kohlensäuren theoretisch berechneten Wertes beträgt. Wir behalten uns die Erweiterung und Berechnung dieser Versuche auf eine folgende Mitteilung vor.

1) H. Euler und G. Lundeqvist, Diese Zeitschrift, Bd. 72, 1911. Bd. 4, 1911.

$\left.{ }^{2}\right)$ H. Euler und A. Fodor, Svenska Vet. Akad. Arkiv f. Kemi, 